\title{
REVISÃO NARRATIVA ABORDANDO A RELAÇÃO ENTRE TDAH E DEPENDÊNCIA DE COCAÍNA
}

\author{
CATEGORIA: Epidemiologia
}

INSTITUIÇÃO: CENTRO UNIVERSITÁRIO SÃO CAMILO

\begin{abstract}
AUTORES: SOUZA, Flávioํㅜ - Avenida Higienópolis 148 apto 163, flaviohaddad45@gmail.com; (11) 996155154

BENEDITO, Vinicius ${ }^{1}$

JÚNIOR, Edegar ${ }^{1}$
\end{abstract}

\section{ORIENTADOR:}

SIMONETTI, Alfredo ${ }^{2}$

${ }^{1}$ Discentes da Faculdade de Medicina do Centro Universitário São Camilo

2 Docente da Faculdade de Medicina do Centro Universitário São Camilo 


\title{
REVISÃO NARRATIVA ABORDANDO A RELAÇÃO ENTRE TDAH E DEPENDÊNCIA DE COCAÍNA
}

\author{
CATEGORIA: Epidemiologia
}

Descritores: Déficit de Atenção e Hiperatividade, Déficit de Atenção, TDAH, Cocaína, Abuso de Cocaína 


\section{RESUMO}

Introdução: O TDAH é um distúrbio neuropsicológico de incidência importante na população, em que os primeiros sintomas se manifestam, tipicamente, na infância. De acordo com a OMS, cerca de 4\% da população mundial adulta apresenta TDAH. Artigos científicos referem uma predominância maior desse distúrbio entre populações com dependências de drogas lícitas e ilícitas, e uma suposta predisposição a dependência entre aqueles que são acometidos por TDAH. O presente estudo visa verificar a veracidade dessa informação e suas possíveis explicações, com enfoque na dependência química de cocaína.

Metodologia: Com base nisso, foi feita uma revisão cruzando os temas 'TDAH'e 'dependência de cocaína' e fizemos a seguinte pergunta como forma de direcionar nosso estudo: 'Há predisposição de pacientes diagnosticados com TDAH a terem maior dependência química de cocaína?'.

Resultados: Um número considerável dos artigos relatou uma incidência elevada de transtornos psiquiátricos nos indivíduos com TDAH e com dependência de cocaína. Quatro estudos transversais apontaram resultados interessantes: há uma prevalência de déficit de atenção e hiperatividade de, respectivamente, $61,25 \%$ em uma unidade terapêutica para álcool e drogas e 18,1\% em uma instituição de detenção de adolescentes com problemas legais contra 5\% na população geral. Ademais, entre os usuários de cocaína $22 \%$ e 14,5\% possuíam critérios diagnósticos para TDAH. Por serem estudos transversais não é possível atribuir causa e efeito aos dados encontrados, mesmo assim são números instigantes. Todos esses dados se relacionam diretamente com o principal sintoma presente nos usuários de cocaína com TDAH: a impulsividade. O tratamento do TDAH nos pacientes dependentes de cocaína se mostrou como uma estratégia eficaz para redução no consumo da droga e na diminuição da abstinência.

Discussão: Existem diversas tentativas de se explicar essa relação estatística entre o Transtorno de Déficit de Atenção e Hiperatividade e a dependência de cocaína. Uma dessas explicações tenta correlacionar o efeito da cocaína no circuito neuronal do portador de TDAH de duas maneiras: A primeira é a de que cocaína mitiga os efeitos neuropsicológicos negativos do TDAH, como inquietação, a segunda é que a cocaína, pelo seu antagonismo na recaptação de dopamina, mimetiza a ação das anfetaminas usadas no tratamento do Déficit. 
Outra explicação utiliza exames de neuroimagens para explicar alterações no sistema de recompensa dos portadores de TDAH. Esses que possuem uma predileção por recompensas imediatas têm uma maior suscetibilidade ao uso de substâncias. A cocaína, em especial, possui singular efeito no cortex frontal, atrapalhando o ordenamento executivo e dificultando o controle inibitório. Reforçando o caráter impulsivo do portador de TDAH.

Conclusão: Existe uma relação estatística importante entre usuários de Cocaína e TDAH, que se explica pela impulsividade dos pacientes com o distúrbio neuropsicológico. Essa impulsividade se exacerba quando estes se tornam dependentes de cocaína. 


\section{CONCEITO E EPIDEMIOLOGIA:}

Déficit de atenção e hiperatividade (TDAH) é um transtorno comportamental que afeta aproximadamente 5\% a 10\% das crianças em todo o mundo (FARAONE et al, 2003) e (BIEDERMAN; FARAONE, 2005). O TDAH se manifesta como um padrão de comportamento que leva a um comprometimento social, educacional e ocupacional significativo. As principais características do transtorno são desatenção, hiperatividade e impulsividade (AMERICAN PSYCHIATRIC ASSOCIATION, 2013). Desde Cantwell (1996), o diagnóstico de TDAH é clínico, baseado em um quadro clínico persistente, de início precoce e que pode levar a significativo prejuízo funcional. $\mathrm{O}$ TDAH deve ser visto como um distúrbio heterogêneo, há uma variação considerável no comportamento, dependendo da tarefa e do estado motivacional do individuo (RUSSELL, 2011).

Oortmerssen et al (2012) apontam que o uso abusivo de cocaína está associado a diferentes comorbidades, como o TDAH; podendo este transtorno estar relacionado ao Transtorno por Uso de Substâncias (TUS) em cerca de 20\% dos indivíduos, independentemente do sexo ou origem étnica.

O vigente estudo visa, através de uma revisão narrativa da literatura científica, ratificar essa relação. 


\section{METODOLOGIA:}

\section{PUBMED e BVS}

Descritores: with Hyperactivity) OR (ADDH)) OR (Attention Deficit Disorder)) OR (Attention Deficit Disorders)) OR (Attention Deficit Disorders with Hyperactivity)) OR (Attention Deficit Hyperactivity Disorder)) OR (Attention Deficit Hyper a ctivity Disorders)) OR (Deficit Disorder, Attention)) OR (Deficit Disorders, Attention)) OR (Deficit-Hyperactivity Disorder, Attention)) AND (Cocaine)) OR (Abuse, Cocaine)) OR (Addiction, Cocaine)) OR (Cocaine Abuse)) OR (Cocaine Addiction)) OR (Cocaine Dependence)) OR (Cocaine Related Disorders)) OR (Dependence, Cocaine)) OR (Dependences, Cocaine)

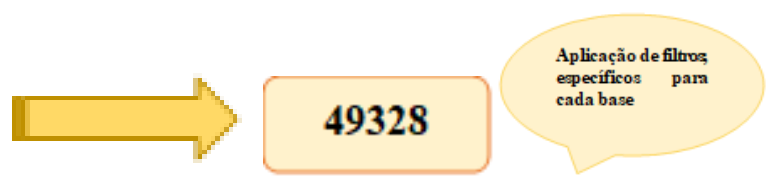

\section{3}

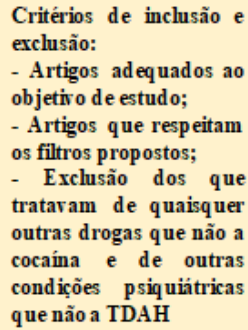

Foi realizada, entre os meses de maio de 2020 e setembro de 2020, uma revisão integrativa de literatura, de fontes primárias e secundárias, com tratamento qualitativo dos resultados encontrados na base de dados PubMed e BVS. Definiu - se área e tema de pesquisa, seguida por criteriosa escolha de descritores e das referências a fazerem parte do estudo, leitura das fontes e análise das mesmas, apresentação das informações de forma compilada e sintética em um quadro sinóptico estruturado para posterior análise, 
contendo: título, autores, ano de publicação, bases de dados, tipo de estudo, local de publicação, bem como principais informações dos artigos estudados.

A combinação dos descritores foi efetuada por meio dos operadores booleanos AND e OR; sendo os filtros usados na PubMed: textos disponíveis gratuitamente e na íntegra; publicações nos últimos 10 anos; apenas ensaios clínicos e realizados em humanos. Já na BVS, os filtros usados foram: textos completos e gratuitos; disponíveis na LILACS; IBECS; BINACIS; MedCarib e Index Psicologia; com os assuntos principais "Cocaína" e "Transtornos Relacionados ao uso de cocaína".

Assim, foram identificados inicialmente 49.328 artigos, dos quais após a aplicação dos filtros usados, restaram 693. Após isso, 677 foram eliminados através da leitura de títulos e seus respectivos resumos, por aplicação dos critérios de inclusão e exclusão. Os critérios de inclusão foram: artigos adequados ao objetivo de estudo; artigos que respeitassem os filtros propostos; análise simultânea de TDAH e dependência por cocaína. Foram excluídos: artigos que não cumpriam os critérios de inclusão; que tratavam da relação do TDAH com demais doenças psíquicas; avaliação de dependência por quaisquer outras drogas que não a por cocaína, como outras heroína, álcool, crack ou opióides. 


\section{DISCUSSÃO}

Partindo pela ótica de análise dos artigos podemos categorizar os resultados em 3 principais grupos. O primeiro grupo composto por artigos em que toda a amostra é dependente de drogas, o segundo grupo formado por artigos em que há uma comparação entre uma amostra dependente de cocaína e outro controle, e o terceiro grupo de artigo em que todos os participantes possuem TDAH. Apesar dos pontos de vista singulares, os resultados confluem. O TDAH possui uma notória prevalência entre usuários de drogas, principalmente cocaína e crack.

As teorias para esse fenômeno são diversas, desde explicações fisiopatológicas, bioquímicas até comportamentais e sociais.

\section{Aspectos genéticos e ambientais do TDAH}

O TDAH possui importante componente hereditário, resultado de complexas interações genéticas e ambientais (FARAONE, 2004) e (THAPAR et al, 2005). Em estudos epigenéticos foram encontradas correlações da doença com polimorfismos de genes que codificam subunidades do receptor de dopamina, com o transportador da dopamina, da serotonina e da proteína SNAP25. (GENRO et al, 2007); (GORNICK et al, 2007). Há também teorias que correlacionam o TDAH com variantes dos genes que codificam a monoamina oxidase A, a dopamina $\beta$-hidroxilase, o transportador de norepinefrina, e o $\alpha$ 2-adrenoceptor (KIM et al, 2006); (BROOKES K et al, 2006); (FARAONE, KHAN, 2006). Além disso, foram encontrados diversos riscos ambientais relacionados ao transtorno, como exposição pré-natal à drogas, complicações obstétricas, traumatismo craniano (BIEDERMAN; FARAONE, 2005) e (ROMANO, 2006).

\section{Vias neurais envolvidas}

Quanto à possíveis anormalidades funcionais, os achados mais consistentes no TDAH são déficits na atividade neural dos circuitos fronto-estriatal e frontoparietal (DICKSTEIN et al, 2006). Já estudos de neuroimagem demonstraram anormalidades funcionais no córtex frontal dorsal e inferior, córtex cingulado anterior, gânglios basais, tálamo e cerebelo de pacientes com TDAH (SCHERES et al, 2007) e (BUSH, 2009).

No trabalho de Scheres et al (2007), a ressonância magnética funcional (fMRI) revelou redução da ativação do estriado ventral em adolescentes com TDAH durante uma 
tarefa de antecipação de recompensa, sugerindo que circuitos neuronais fronto-estriados relacionados à recompensa estão prejudicados do ponto de vista funcional; além da disfunção executiva pré-frontal comumente observadas.

Aumentos no DAT estriatal de até $70 \%$ foram encontrados em crianças e adultos com TDAH (CHEON et al, 2003), o que sugere que o DAT1 (gene) pode estar superexpresso no corpo estriado de indivíduos com TDAH, resultando em redução da dopamina em fenda sináptica. No entanto, nem todos os estudos encontraram DAT aumentado nesses pacientes, como no estudo de Jucaite et al (2005) por exemplo; e descobertas mais recentes ainda, sugerem que em alguns adultos que nunca usaram drogas e são portadores de TDAH, os níveis de DAT no caudado esquerdo e no núcleo accumbens são reduzidos (VOLKOW et al, 2007).

\section{Teoria dopaminérgica}

Há evidências convincentes para sugerir que os sintomas de TDAH podem resultar de função prejudicada da dopamina no cérebro, especificamente desenvolvimento mediado pela dopamina e monitoramento do comportamento motivado e formação de memória relacionada à recompensa. A liberação deficiente de dopamina durante o desenvolvimento, pode prejudicar o fortalecimento das conexões sinápticas relacionadas à recompensa e enfraquecer a associação de pistas preditivas com o resultado e comportamento de produção de recompensa. Como consequência direta disso, um indivíduo com TDAH pode ser incapaz de estabelecer sequências longas ou complicadas de comportamento, em resposta a padrões temporais específicos de apresentação de estímulos de predição de recompensa (SAGVOLDEN et al, 2005); (JOHANSEN et al, 2009).

Fillmore e Rush (2002) compartilham desses conceitos, à medida em que apontam que os usuários de cocaína com TDAH podem apresentar prejuízos na autorregulação comportamental; devido à ativação dopaminérgica repetitiva dos circuitos neurais, em função do uso repetidamente frequente de cocaína; pois essa droga ao inibir vias neurais específicas, leva à perda de controle sobre os comportamentos impulsivos, incluindo a autoadministração de cocaína.

Os distúrbios dopaminérgicos são capazes de explicar mudanças importantes na motivação, que por sua vez, justificariam a busca por recompensa e a aversão a recompensas atrasadas (WILBERTZ et al, 2012). 


\section{Teoria catecolaminérgica}

Entretanto, a teoria da dopamina não é a única a buscar explicações para esse fenômeno, pois Russell (2011) discorre sobre as alterações nas vias catecolaminérgicas, que envolvem também o nucleus accumbens, com prejuízo da atenção seletiva e do sistema de recompensa; o que pode estar associado a um maior risco de uso e dependência de substâncias, segundo Galéra, et al (2013).

\section{Funções neuropsicológicas}

A teoria proposta no estudo de Barkley (1997), sustenta que o desenvolvimento satisfatório da inibição, é essencial para o desempenho normal de cinco outras habilidades neuropsicológicas: memória operacional, internalização da fala, autorregulação da motivação afetiva - excitação, reconstituição e controle motor - fluência - sintaxe. Dentre essas funções, as quatro primeiras são consideradas de natureza executiva porque permitem a autorregulação, o controle do comportamento por informações representadas internamente e a organização temporal do comportamento. Essa autorregulação dá origem à direção e à persistência do comportamento em direção a objetivos futuros e à capacidade de voltar a engajar esse comportamento, se for interrompido.

Portanto, a inibição comportamental está ligada à memória de trabalho e sentido de tempo, internalização, automotivação, criatividade comportamental e autocontrole de maneira geral, tendo aplicação imediata aqui para a compreensão do TDAH, pois sugerese que uma falha na inibição comportamental leve aos sintomas predominantes do TDAH: hiperatividade, desatenção, distração e impulsividade. Evidências apontam para uma deficiência em três processos que envolvem a inibição comportamental no TDAH: inibição de respostas prepotentes, interrupção de respostas contínuas com feedback sobre erros e controle de interferência. Quando o modelo funções executivas discutido acima é estendido ao TDAH, são previstos prejuízos nas quatro funções executivas em quem tem esse transtorno (BARKLEY, 1997).

Esses déficits executivos criam, então, deficiências no controle motor-fluênciasintaxe e no controle do comportamento motor. Os resultados das pesquisas sobre o TDAH, em vários graus, parecem ser consistentes com os déficits nos componentes do modelo. Grande parte da literatura existente sobre os déficits cognitivos ou neuropsicológicos no TDAH sofre de vários problemas metodológicos. $\mathrm{O}$ mais significativo entre eles teria de ser (a) o uso de tamanhos de amostra tão pequenos que há poder estatístico inadequado para detectar os tamanhos de efeito pequenos a moderados 
que estão provavelmente associados a déficits nessas funções executivas; o uso de critérios de seleção inconsistentes entre os estudos na definição de TDAH; a falha no controle de transtornos comórbidos potencialmente confusos; a falta de atenção aos efeitos maturacionais e de gênero; e a falta de consideração pelos efeitos da história familiar de TDAH sobre os déficits associados ao TDAH em crianças. Tais compromissos processuais tornam grande parte da pesquisa existente inadequada para testar $\mathrm{e}$ potencialmente falsificar as previsões do presente modelo. Uma pesquisa mais bem elaborada deve ajudar a resolver essas inconsistências e, sem dúvida, levará a modificações do modelo aqui apresentado (BARKLEY, 1997).

Crunelle et al (2013) dizem que a atenção e memória de trabalho entre indivíduos com TDAH e uso de cocaína são as funções centrais em sua psicopatologia, pois os dependentes de cocaína com TDAH apresentam níveis significativamente mais elevados de impulsividade motora e cognitiva. Assim, para Szobot e Romano (2007), o uso de cocaína interfere negativamente no prognóstico dos pacientes com TDAH e adicção de cocaína concomitantes, pois esses indivíduos cursam com recidivas mais frequentes e menor adesão terapêutica de ambas as condições clínicas. Além disso, apontam que o controle dos impulsos motores e cognitivos piora quando ambos os transtornos estão associados; isso porque o controle inibitório depende do bom funcionamento dos lobos frontais, os quais estão relacionados a vários subtipos de inibição; especulando que esses indivíduos precisam de mais - ou talvez sobrepostos - circuitos no lobo frontal, para que o controle de impulso adequado possa ser alcançado. Portanto, os indivíduos com TDAH e dependência de cocaína são mais impulsivos do que os indivíduos sem uso de cocaína devido a seus circuitos executivos disfuncionais e de recompensa / motivacional.

Outros autores concordam com isso, como Cobos et al (2011), ao dizer que usuários de cocaína com TDAH que procuram tratamento sentem maior fissura durante os dias após o início do tratamento, permanecendo abstinentes por um período mais curto, daí maior recidiva e menor adesão. Os resultados encontrados até aqui, estão de acordo com as evidências apontadas por Balconi et al (2014) e Volkow et al (2010) sobre a associação entre o TDAH e o uso de cocaína; à medida em que tal associação pode ser explicada por mecanismos atuantes no sistema de recompensa, como a menor atuação de receptores de dopamina.

Em testes de neuroimagem de pacientes com TDAH, em estudo proposto por Wilbertz et al (2012), a dissociação neural é mostrada entre as decisões sobre recompensa imediata e adiada e hipoativação orbitofrontal. Assim, esses indivíduos 
buscariam situações mais gratificantes e isso pode estar diretamente associado ao uso de cocaína, conforme apontado na literatura (BALCONI et al 2014) e (VOLKOW et al 2010).

Portanto, o TDAH em adultos também está associado a múltiplos déficits das funções executivas, como memória de trabalho, processamento emocional, processamento temporal e controle inibitório. Como o TDAH é considerado um transtorno do desenvolvimento, espera-se que a maioria desses sintomas diminua na vida adulta. Os sintomas persistentes, parecem estar relacionados à faceta da impulsividade; essa que é uma característica central do TDAH. Essa impulsividade tem correlação com erros de controle inibitório, que apresentam déficits em usuários de substâncias (FILLMORE; RUSH, 2002). 


\section{RESULTADOS:}

O estudo de Camargo, et al (2016), se desenvolveu a partir da seleção de 80 pacientes (53 homens e 27 mulheres) internados entre julho de 2013 e dezembro de 2014 em dois centros de reabilitação para dependentes químicos. Os critérios de inclusão foram: ter história de uso de drogas; estar em um centro de reabilitação; e ter 18 anos ou mais. Os critérios de exclusão foram: recusa em assinar o termo de consentimento livre e esclarecido e incapacitação cognitiva e comportamental. A lista de verificação de sintomas da Adult ADHD SelfReport Scale (ASRS-18) foi aplicada para qualificar e quantificar o TDAH, principalmente por meio do acesso à frequência dos sintomas de TDAH.

Dos 80 pacientes, $49(61,25 \%)$ foram classificados com achados clínicos sugestivos de TDAH. 72 pacientes eram dependentes de múltiplas drogas mas não houve diferença estatística entre as populações com e sem TDAH $(p=0,0501)$. As drogas com maior e menor número de usuários foram álcool e cocaína, respectivamente. A cocaína apresentou o maior percentual de usuários dependentes. Dos pacientes que tiveram pelo menos contato ocasional com cocaína, 77,78\% desenvolveram dependência, enquanto para as demais drogas o valor correspondente foi de 40,5\% (p <0,0001). A idade de início do uso de cocaína e seus derivados foi menor na população com TDAH ( $\mathrm{p}=0,033)$, assim como a idade de admissão à reabilitação $(\mathrm{p}=0,004)$ (CAMARGO, et al 2016).

Apesar dessa aparente falta de associação entre TDAH e a gravidade do uso de substâncias no presente estudo, houve uma diferença significativa na idade do primeiro uso de cocaína entre os grupos com e sem TDAH. Corroborando esse achado, Dunne et al (2014) encontraram menor idade para o primeiro uso de cocaína entre pacientes com TDAH em um estudo com 941 usuários de substâncias. Nos pacientes com TDAH em comunidades terapêuticas, uma idade mais baixa no primeiro uso de cannabis em pacientes com TDAH parece levar a um uso mais pesado de cocaína; essas correlações são específicas para o grupo com TDAH, uma vez que não foram identificadas no grupo sem TDAH ou no grupo geral (CAMARGO et al, 2016).

O estudo encontrou uma correlação inversa entre a frequência de sintomas de TDAH e a idade ao primeiro uso de todas as substâncias estudadas. Isso pode indicar que, entre os pacientes tratados em comunidades terapêuticas, aqueles com sintomas de TDAH mais comuns podem começar a cocaína mais cedo do que os pacientes sem nenhum ou menos sintomas de TDAH. 
Apesar de ser a droga a que menos pacientes apresentavam histórico de exposição, a cocaína causou mais dependência (transtorno por uso de substâncias) nos pacientes investigados. Embora não tenha havido diferença no percentual de usuários que se tornaram dependentes entre as populações com e sem TDAH, o fato de os pacientes com TDAH terem contato precoce com cocaína os torna mais suscetíveis à dependência em uma idade mais jovem, o que por sua vez pode significar que eles são admitidos para reabilitação mais cedo. Nessa amostra, houve alta prevalência de TDAH em comparação com a população em geral. Descobriu-se que os indivíduos com TDAH apresentam padrões diferentes de comportamento de dependência de drogas. Essas características podem ser explicadas pelo contato mais precoce com a droga que mais causava dependência, a cocaína. (CAMARGO et al, 2016).

O objetivo do estudo de Cancian ACM et al (2017), foi analisar a relação entre o uso de cocaína e o diagnóstico de transtorno do déficit de atenção e hiperatividade. Uma amostra composta por 971 participantes, todos adultos, com idade entre 18 e 59 anos foi dividida em dois grupos: 1) grupo de usuários de cocaína $(n=407)$ e 2) grupo de nãousuários de cocaína $(n=564)$. Os critérios de inclusão para o primeiro foram: internação em serviços de saúde públicos e / ou privados especializados que atendem pacientes da capital Porto Alegre, RS, Brasil e Região Metropolitana; estar entre os $7^{\circ}$ e $15^{\circ}$ dia de abstinência de substâncias psicoativas; ter completado pelo menos cinco anos de escolaridade formal e relatar o uso de cocaína como fator que motivou a busca por internação. O grupo não clínico foi composto por pacientes residentes no Estado do Rio Grande do Sul (Brasil), principalmente na Região Metropolitana de Porto Alegre. Os dados foram coletados por psicólogos e graduandos de psicologia. Já o grupo clínico foi composto por indivíduos atendidos em ambulatórios especializados no tratamento de dependência química. Foram encontradas diferenças significativas na presença do diagnóstico de transtorno de déficit de atenção e hiperatividade ( $\mathrm{p}=0,001)$; como problemas de atenção $(\mathrm{p}=0,001)$, que podem estar relacionados a mudanças associadas à busca por situações gratificantes, como é o uso de substâncias. Os resultados mostram uma associação significativa entre as duas condições, confirmando os achados da literatura. Várias hipóteses estão envolvidas na relação entre TDAH e cocaína, incluindo maior sensibilidade no sistema de recompensa, visto que indivíduos com TDAH teriam menos receptores de dopamina. Assim, seriam provocadas mudanças na motivação e na busca por situações gratificantes e na impulsividade, característica de ambos os transtornos. 
O estudo de Ribas-Siñol et al (2015) é descritivo, e aborda a relação entre o uso de drogas e as características clínicas, demográficas e criminais em uma amostra de 144 jovens. $18,1 \%$ apresentam TDAH e 78,5\% eram consumidores de drogas; $51,4 \%$ do total consumiam apenas 1 substância. Há uma tendência entre os adolescentes com TDAH a consumirem cocaína.

Vergara-Moragues et al. (2011) realizou um estudo transversal com uma amostra de 166 pacientes de seis comunidades terapêuticas para dependência química. O estudo tinha como critérios de inclusão 1) ser dependente de cocaína por no mínimo 12 meses 2) ter pelo menos 18 anos, 3) ser alfabetizado, 4) ter assinado o termo de consentimento. Os critérios de exclusão foram: 1) presença de déficit cognitivo orgânico que impediu exame psíquico, 2) processo criminal que impedisse o seguimento na Comunidade pelo tempo de análise. Foram realizados três questionários sob supervisão e avaliação de psiquiatras. Inicialmente o CAADID (Conners' Adult ADHD Diagnostic Interview for the DSMIV) para diagnóstico de TDAH, o PRISM-IV (Psychiatric Research Interview for substance and Mental Disorders) para avaliar comorbidades psiquiátricas e dependência química e o Current Behavior Scale Self-Report by Barkley para avaliar função executiva. Após o primeiro instrumento, os participantes foram divididos em dois grandes subgrupos. Os de dependentes de cocaína com diagnóstico de TDAH e o dos dependentes sem o diagnóstico. $91 \%$ de toda amostra era composta por homens, $59 \%$ solteiros, $86,1 \%$ possuíam ensino básico completo apenas. 66,3\% eram desempregados, 61,4\% tinham alguma ficha criminal, apenas 18,1\% tinham algum diagnóstico prévio. $14,5 \%$ da amostra foi diagnosticada com TDAH, sendo que nenhum desses possuía algum tratamento prévio para o transtorno. O tipo hiperativo foi o mais comum, presente em $45 \%$ dos portadores de TDAH. O Deficit de Atenção e Hiperatividade foi o transtorno mais prevalente na amostra, seguido pelo transtorno antissocial com $6 \%$ da amostra. Em uma análise comparativa, o grupo com TDAH possuía importante comprometimento da função executiva como tomada de decisão impulsiva, fácil distração, dificuldade em realizar tarefas cadenciadas, propensão em dirigir em altas velocidade, dificuldade em respeitar compromissos. (Vergara-Moraques, 2011)

Carmen Silvia (2014) realizou também um estudo transversal observacional comparando dois grupos, um de portadores de TDAH e dependentes de cocaína/crack e outro composto por portadores apenas de TDAH. Seus critérios de inclusão foram: idade entre 18-60 anos, ensino básico completo, QI maior que 80, sem uso medicações prévias para o TDAH e no grupo de usuários, estar abstinente por pelo menos 15 dias. Foram 
aplicados questionários sociodemográficos e clínicos como o $\quad$ 1) ARS 18 (Adult Self Report) para avaliação do TDAH, 2) M.I.N.I 500 para detectar comorbidades psiquiátricas, 3) ASSIST que é uma triagem sobre o uso de álcool, cigarro e outras substancias, 4) ASI-6 também para uso de drogas, 5) BIS-11 para análise de impulsividade. A amostra foi composta principalmente por homens. $\mathrm{O}$ grupo dependente de cocaína possuía pior desempenho escolar e eram mais socialmente vulneráveis, além de terem mais risco para suicídio. Transtorno de personalidade antissocial, depressão maior e episódio maníaco eram mais prevalentes no grupo dependente de cocaína/crack. A primeira droga usada nos dois grupos foi a nicotina, sendo iniciada mais precocemente no grupo de usuários. A média de QI do grupo dependente químico foi menor que o grupo apenas com Deficit de Atenção. (Carmen Silvia, 2014)

Restrepo-Bernal et al (2014) também conduziu um estudo observacional transversal com uma análise de casos e controles. Em uma amostra de 107 casos e 340 controles entre 13 e 18 anos, a autora utilizou do CIDI-OMS para o diagnóstico de transtornos psiquiátricos. Dos resultados encontrados, 59,1\% eram mulheres, em média com 16 anos, 86,1\% tinham ensino médio completo. 6,3\% dos adolescentes possuíam critérios para TDAH, 31,82\% das mulheres e 12,57\% dos homens tinham comportamento suicida. Dentro do grupo com ideação suicida, a incidência de TDAH foi maior. (RESTREPO-BERNAL, 2014)

Frances R. Levin et al (2017) conduziu um importante estudo duplo-cego, randomizado, onde foram separados 2 grupos de indivíduos com TDAH e dependentes de cocaína, tendo duração de 14 semanas. Uma parte do grupo recebeu um placebo (sendo este o grupo controle) e o outro recebeu MAS XR (60 ou 80mg diário). O intuito do estudo foi ver o êxito na abstinência da cocaína ao longo do tempo, comparando os dois grupos. Os critérios de inclusão do estudo foram ter entre 18 e 60 anos, ter diagnóstico de dependência de cocaína e critérios diagnósticos de TDAH. Já os critérios de exclusão foram a presença de outras doenças psiquiátricas e presença de alguma outra condição médica. A AISRS score era usado para indicar se houve melhora do TDAH, e os participantes faziam o score a cada duas semanas. Uma diminuição de $30 \%$ nesse score era registrado com melhora no TDAH. O Perfil dos participantes eram de homens, metade caucasianos e metade composta por afro-americanos e hispânicos. Sobre os resultados do estudo, foi observado que a probabilidade de alcançar abstinência de cocaína no grupo em uso de anfetaminas era 4x maior. Ademais, a principal correlação 
observada no estudo foi a seguinte: dentre os pacientes que tiveram melhora na abstinência e no TDAH, a melhora no TDAH costumava acontecer antes.

Marc E. Mooney et al (2015) conduziu um estudo verificando a eficácia do lisdexanfetamina (LDX), vendida comercialmente como Vynvanse, em indivíduos dependentes de cocaína. Este é o primeiro estudo a avaliar a tolerância, eficácia e segurança do LDX. Durante as 14 semanas, parte do grupo recebeu 70mg (dose máxima indicada pela Food and Drug Administration) de LDX, e a outra recebeu placebo apenas. Os critérios de inclusão foram idade entre 18 e 65 anos de idade, condição de saúde mental/psiquiátrica razoável, ECG normal, ausência de histórico de doenças cardiovasculares e correspondência com os critérios do DSM-4 de dependência de cocaína. Já os critérios de exclusão foram transtornos de humor, transtornos psicóticos, transtornos de ansiedade, TDAH e dependência de outras drogas que não sejam marijuana ou nicotina. Sobre os achados do estudo, o grupo que fez uso do LDX relatou importantes efeitos-colaterais, como cefaléia, náusea, vômito, diarreia e ansiedade. No estudo, não foi encontrado um menor uso de cocaína no grupo que recebeu anfetaminas. No entanto, os membros desse grupo relataram ter um impulso muito menor para consumir a droga. Não foi observado diferença de frequência cardíaca, pressão arterial e peso entre os grupos. 


\section{CONCLUSÃO:}

$\mathrm{Na}$ interpretação dos resultados, devem ser consideradas as limitações da pesquisa, com o desenho de estudo. Portanto, nenhuma relação de causa e efeito pode ser atribuída entre o diagnóstico de TDAH e o uso de cocaína, mas sim inferências que se referem a uma associação significativa. Diante desses aspectos, sugere-se pesquisas que investiguem o tema longitudinalmente e utilizem outros recursos de pesquisa, como a neuroimagem; marcadores

substâncias psicoativas, visto que comorbidades como o TDAH podem interferir no prognóstico e na tentativa de interromper o uso de cocaína.

Assim, investigar o TDAH em usuários de cocaína é fundamental para compreender o indivíduo, suas dificuldades e características, que fazem parte do processo de desintoxicação e reabilitação. É interessante que os profissionais de saúde consigam identificar essas associações para orientar efetivamente o tratamento do usuário. 


\section{REFERÊNCIAS:}

Faraone, SV, Sergeant, J., Gillberg, C., e Biederman, J. 2003. A prevalência mundial de TDAH: é uma condição americana? Psiquiatria Mundial 2: 104-113.

BIEDERMAN, Joseph; FARAONE, Stephen V. Attention-deficit hyperactivity disorder. The Lancet, [S.L.], v. 366, n. 9481, p. 237-248, jul. 2005. Elsevier BV. http://dx.doi.org/10.1016/s0140-6736(05)66915-2.

American Psychiatry Association. Manual diagnóstico e estatístico de transtornos mentais. $5^{\mathrm{a}}$ ed. Washington, DC: American Psychiatric Association; 2013. (AMERICAN PSYCHIATRIC ASSOCIATION, 2013)

CANTWELL, Dennis P.. Attention Deficit Disorder: a review of the past 10 years. Journal Of The American Academy Of Child \& Adolescent Psychiatry, [S.L.], v. 35, n. 8, p. 978-987, ago. 1996. Elsevier BV. http://dx.doi.org/10.1097/00004583-199608000-00008.

RUSSELL, Vivienne Ann. Overview of Animal Models of Attention Deficit Hyperactivity Disorder (ADHD). Current Protocols In Neuroscience, [S.L.], v. 54, n. 1, p. 1-25, jan. 2011. Wiley. http://dx.doi.org/10.1002/0471142301.ns0935s54.

OORTMERSSEN, Katelijne van Emmerik-Van; GLIND, Geurt van de; BRINK, Wim van Den; SMIT, Filip; CRUNELLE, Cleo L.; SWETS, Marije; SCHOEVERS, Robert A.. Prevalence of attention-deficit hyperactivity disorder in substance use disorder patients: a meta-analysis and meta-regression analysis. Drug And Alcohol Dependence, [S.L.], v. 122, n. 1-2, p. 11-19, abr. 2012. Elsevier BV. http://dx.doi.org/10.1016/j.drugalcdep.2011.12.007.

CAMARGO, Carlos Henrique Ferreira; DORNELLES, Tarcísio Fanha; BARSZCZ, Karin; MARTINS, Eduardo Antunes. Attention deficit hyperactivity disorder and drug addiction rehabilitation patients. Arquivos de Neuro-Psiquiatria, [S.L.], v. 74, n. 12, p. 1003-1007, dez. 2016. FapUNIFESP (SciELO). http://dx.doi.org/10.1590/0004$282 \times 20160163$.

DUNNE, Eugene M.; HEARN, Lauren E.; ROSE, Jonathan J.; LATIMER, William W.. ADHD as a risk factor for early onset and heightened adult problem severity of illicit substance use: an accelerated gateway model. Addictive Behaviors, [S.L.], v. 39, n. 12, p. 1755-1758, dez. $2014 . \quad$ Elsevier BV. http://dx.doi.org/10.1016/j.addbeh.2014.07.009.

Vergara-Moragues E, González-Saiz F, Lozano Rojas O, Bilbao Acedos I, Fernández Calderón F, Betanzos Espinosa P, Verdejo García A, Pérez García M: Diagnosing Adult Attention Deficit/Hyperactivity Disorder in Patients with Cocaine Dependence: Discriminant Validity of Barkley Executive Dysfunction Symptoms. Eur Addict Res 2011;17:279-284. doi: 10.1159/000329725

MIGUEL, Carmen Silvia. Estudo comparativo do desempenho cognitivo de portadores adultos do Transtorno de Déficit de Atenção Hiperatividade (TDAH) associado a 
Transtorno por Uso de Substâncias Psicoativas (TUSP) e portadores adultos de TDAH sem a presença de TUSP. 2014. Dissertação (Mestrado em Psiquiatria) - Faculdade de Medicina, University of São Paulo, São Paulo, 2014. doi:10.11606/D.5.2014.tde27082014-141353. Acesso em: 2020-11-11.

RESTREPO-BERNAL,

Diana

et al

. Suicidal Behavior and Attention Decifit Hyperactivity Disorder in Adolescents of Med ellin (Colombia), 2011-2012. rev.colomb.psiquiatr., Bogotá , v. 43, n. 4, p. 186193, Oct. 2014

- Available from <http://www.scielo.org.co/scielo.php?script=sci_arttext\&pid=S0034$74502014000400003 \& \operatorname{lng}=$ en\&nrm=iso $>$. access on 11 Nov. 2020

FARAONE, Stephen V. Genetics of adult attention-deficit/hyperactivity disorder. Psychiatric Clinics Of North America, [S.L.], v. 27, n. 2, p. 303-321, jun. 2004. Elsevier BV. http://dx.doi.org/10.1016/s0193-953x(03)00090-x.

THAPAR, Anita; O'DONOVAN, Michael; OWEN, Michael J. The genetics of attention deficit hyperactivity disorder. Human Molecular Genetics, [S.L.], v. 14, n. 2, p. 275282, 15 out. 2005. Oxford University Press (OUP). http://dx.doi.org/10.1093/hmg/ddi263.

GENRO, Júlia P.; ZENI, Cristian; POLANCZYK, Guilherme V.; ROMAN, Tatiana; ROHDE, Luis A.; HUTZ, Mara H.. A promoter polymorphism $(-839 \mathrm{C}>\mathrm{T})$ at the dopamine transporter gene is associated with attention deficit/hyperactivity disorder in Brazilian children. American Journal Of Medical Genetics Part B: Neuropsychiatric

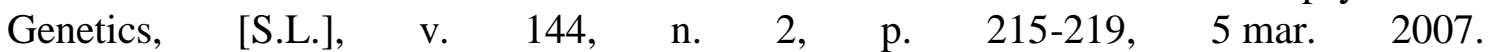
Wiley. http://dx.doi.org/10.1002/ajmg.b.30428.

GORNICK, M.C.; ADDINGTON, Anjene; SHAW, P.; BOBB, A.J.; SHARP, W.; GREENSTEIN, D.; AREPALLI, S.; CASTELLANOS, F.X.; RAPOPORT, J.L.. Association of the dopamine receptor D4 (DRD4) gene 7-repeat allele with children with attention-deficit/hyperactivity disorder (ADHD): an update. American Journal Of Medical Genetics Part B: Neuropsychiatric Genetics, [S.L.], v. 144, n. 3, p. 379-382, 2007. Wiley. http://dx.doi.org/10.1002/ajmg.b.30460.

KIM, C.-H.; HAHN, M. K.; JOUNG, Y.; ANDERSON, S. L.; STEELE, A. H.; MAZEIROBINSON, M. S.; GIZER, I.; TEICHER, M. H.; COHEN, B. M.; ROBERTSON, D.. A polymorphism in the norepinephrine transporter gene alters promoter activity and is associated with attention-deficit hyperactivity disorder. Proceedings Of The National Academy Of Sciences, [S.L.], v. 103, n. 50, p. 19164-19169, 4 dez. 2006. Proceedings of the National Academy of Sciences. http://dx.doi.org/10.1073/pnas.0510836103.

BROOKES, K., Xu, X., Chen, W., Zhou, K., Neale, B., Lowe, N., Anney, R., Franke, B., Gill, M., Ebstein, R., Buitelaar, J., Sham, P., Campbell, D., Knight, J., Andreou, P., Altink, M., Arnold, R., Boer, F., Buschgens, C., Butler, L., Christiansen, H., Feldman, L., Fleischman, K., Fliers, E., Howe-Forbes, R., Goldfarb, A., Heise, A., Gabriels, I., KornLubetzki, I., Johansson, L., Marco, R., Medad, S., Minderaa, R., Mulas, F., Muller, U., Mulligan, A., Rabin, K., Rommelse, N., Sethna, V., Sorohan, J., Uebel, H., Psychogiou, L., Weeks, A., Barrett, R., Craig, I., Banaschewski, T., Sonuga-Barke, E., Eisenberg, J., Kuntsi, J., Manor, I., McGuf fi n, P., Miranda, A., Oades, RD, Plomin, R., Roeyers, 
H., Rothenberger, A., Sergeant, J., Steinhausen, HC, Taylor, E., Thompson, M., Faraone, SV e Asherson, P. 2006. A análise de 51 genes no transtorno de déficit de atenção e hiperatividade do tipo combinado DSM-IV: Sinais de associação em DRD4, DAT1 e 16 outros genes. Mol. Psiquiatria 11: 934-953.

FARAONE, SV e Khan, SA 2006. Gene candidato estudos de transtorno de déficit de atenção / hiperatividade. J. Clin. Psiquiatria 67: 13-20.

ROMANO, E.. Development and Prediction of Hyperactive Symptoms From 2 to 7 Years in a Population-Based Sample. Pediatrics, [S.L.], v. 117, n. 6, p. 2101-2110, 1 jun. 2006. American Academy of Pediatrics (AAP). http://dx.doi.org/10.1542/peds.2005-0651.

DICKSTEIN, Steven G.; BANNON, Katie; CASTELLANOS, F. Xavier; MILHAM, Michael P.. The neural correlates of attention deficit hyperactivity disorder: an ale metaanalysis. Journal Of Child Psychology And Psychiatry, [S.L.], v. 47, n. 10, p. 10511062, nov. 2006. Wiley. http://dx.doi.org/10.1111/j.1469-7610.2006.01671.x.

Scheres, A., Milham, MP, Knutson, B., and Castellanos, $\quad$ FX 2007. Hiporresponsividade do estriado ventral durante a antecipação da recompensa no transtorno de déficit de atenção / hiperatividade. Biol. Psiquiatria 61: 720-724

BUSH, George. Attention-Deficit/Hyperactivity Disorder and Attention Networks. Neuropsychopharmacology, [S.L.], v. 35, n. 1, p. 278-300, 16 set. 2009. Springer Science and Business Media LLC. http://dx.doi.org/10.1038/npp.2009.120.

CHEON, Keun-Ah; RYU, Young Hoon; KIM, Young-Kee; NAMKOONG, Kee; KIM, Chan-Hyung; LEE, Jong. Dopamine transporter density in the basal ganglia assessed with [123I]IPT SPET in children with attention deficit hyperactivity disorder. European Journal Of Nuclear Medicine And Molecular Imaging, [S.L.], v. 30, n. 2, p. 306311, fev. 2003. Springer Science and Business Media LLC. http://dx.doi.org/10.1007/s00259-002-1047-3.

JUCAITE, Aurelija; FERNELL, Elisabeth; HALLDIN, Christer; FORSSBERG, Hans; FARDE, Lars. Reduced midbrain dopamine transporter binding in male adolescents with attention-deficit/hyperactivity disorder: association between striatal dopamine markers and motor hyperactivity. Biological Psychiatry, [S.L.], v. 57, n. 3, p. 229-238, fev. 2005. Elsevier BV. http://dx.doi.org/10.1016/j.biopsych.2004.11.009.

VOLKOW, Nora D.; WANG, Gene-Jack; NEWCORN, Jeffrey; FOWLER, Joanna S.; TELANG, Frank; SOLANTO, Mary V.; LOGAN, Jean; WONG, Christopher; MA, Yeming; SWANSON, James M.. Brain dopamine transporter levels in treatment and drug naïve adults with ADHD. Neuroimage, [S.L.], v. 34, n. 3, p. 1182-1190, fev. 2007. Elsevier BV. http://dx.doi.org/10.1016/j.neuroimage.2006.10.014.

Mill, J., Sagvolden, T., e Asherson, P. 2005. Seanálise de sequência de Drd2, Drd4 e Dat1 em cepas de ratos SHR e WKY. Behav. Brain Funct. 1:24

Johansen, EB, Killeen, PR, Russell, VA, Tripp, G., Wickens, JR, Tannock, R., Williams, J. e Sagvolden, $\quad$ T. 2009.2 Origins altered reforcement effects inADHD. Behav. BrainFunct. 5: 7. 
FILLMORE, Mark T; RUSH, Craig R. Impaired inhibitory control of behavior in chronic cocaine users. Drug And Alcohol Dependence, [S.L.], v. 66, n. 3, p. 26273, maio 2002. Elsevier BV. http://dx.doi.org/10.1016/s0376-8716(01)00206-x.

WILBERTZ, Gregor; VAN ELST, Ludger Tebartz; DELGADO, Mauricio R.; MAIER, Simon; FEIGE, Bernd; PHILIPSEN, Alexandra; BLECHERT, Jens. Orbitofrontal reward sensitivity and impulsivity in adult attention deficit hyperactivity disorder. Neuroimage, [S.L.], v. 60, n. 1, p. 353-361, mar. 2012. Elsevier BV. http://dx.doi.org/10.1016/j.neuroimage.2011.12.011.

GALÉRA, Cédric; PINGAULT, Jean-Baptiste; FOMBONNE, Eric; MICHEL, Grégory; LAGARDE, Emmanuel; BOUVARD, Manuel-Pierre; MELCHIOR, Maria. Attention Problems in Childhood and Adult Substance Use. The Journal Of Pediatrics, [S.L.], v. 163, n. 6, p. 1677-1683, dez. 2013. Elsevier

BV. http://dx.doi.org/10.1016/j.jpeds.2013.07.008.

BARKLEY, Russell A.. Behavioral inhibition, sustained attention, and executive functions: constructing a unifying theory of adhd.. Psychological Bulletin, [S.L.], v. 121, n. 1, p. 65-94, jan. 1997. American Psychological Association (APA). http://dx.doi.org/10.1037/0033-2909.121.1.65.

CRUNELLE, Cleo L.; VELTMAN, Dick J.; OORTMERSSEN, Katelijne van EmmerikVan; BOOIJ, Jan; BRINK, Wim van Den. Impulsivity in adult ADHD patients with and without cocaine dependence. Drug And Alcohol Dependence, [S.L.], v. 129, n. 1-2, p. 18-24, abr. 2013. Elsevier BV. http://dx.doi.org/10.1016/j.drugalcdep.2012.09.006.

Szobot CM, RomanoM.Co-ocorrênciaentretranstorno de déficit de atenção / hiperatividade e uso de substâncias psicoativas. ADOLEC-Coocorrência entre transtorno de déficit de atenção e hiperatividade e substâncias psicoativas; J Bras Psiquiatr. Janeiro de 2007; 56 (1 Suplemento): 39-44. Português.

COBOS, José Pérez de Los; SIÑOL, Núria; PUERTA, Carmen; CANTILLANO, Vanessa; ZURITA, Cristina López; TRUJOLS, Joan. Features and prevalence of patients with probable adult attention deficit hyperactivity disorder who request treatment for cocaine use disorders. Psychiatry Research, [S.L.], v. 185, n. 1-2, p. 205-210, jan. 2011. Elsevier BV. http://dx.doi.org/10.1016/j.psychres.2009.03.019.

BALCONI, Michela; FINOCCHIARO, Roberta; CAMPANELLA, Salvatore. Reward Sensitivity, Decisional Bias, and Metacognitive Deficits in Cocaine Drug Addiction. Journal Of Addiction Medicine, [S.L.], v. 8, n. 6, p. 399-406, 2014. Ovid Technologies (Wolters Kluwer Health). http://dx.doi.org/10.1097/adm.0000000000000065.

VOLKOW, N D; WANG, G-J; NEWCORN, J H; KOLLINS, S H; WIGAL, T L; TELANG, F; FOWLER, J s; GOLDSTEIN, R Z; KLEIN, N; LOGAN, J. Motivation deficit in ADHD is associated with dysfunction of the dopamine reward pathway. Molecular Psychiatry, [S.L.], v. 16, n. 11, p. 1147-1154, 21 set. 2010. Springer Science and Business Media LLC. http://dx.doi.org/10.1038/mp.2010.97. 